\title{
DISCUSSION
}

\section{On the application of the Hiley formula in driving long piles}

\author{
T. TRIANTAFYLLIDIS (2001). Géotechnique 51, No. 10,891-895
}

\begin{abstract}
K. S. Li, Victor Li \& Associates Ltd/University of Hong Kong

It has long been recognised that the Hiley formula is not valid for long piles (Cornfield, 1961; GEO, 1996). The author has introduced an interesting and useful concept in which the effective length of a pile is related to the time of separation between the hammer and pile after impact. In deriving the effective pile length, the author has relied on the following information or assumptions:
\end{abstract}

(a) information on the maximum compressive stress, $\sigma_{0}$, induced at the pile during driving

(b) an estimate of the average shaft friction along the pile (c) the assumption that $\varepsilon=1$.

In the illustrative example presented in the paper, the values of $\sigma_{0}$ were obtained from the results of pile dynamic analyser (PDA) tests presented in Lam et al. (1994). The average shaft friction was estimated from an empirical correlation with SPT $N$ values, although the general validity of such a correlation has yet to be demonstrated for Hong Kong soils. The assumption of $\varepsilon=1$ can only be regarded as an approximation, not a proven fact.

Experience in Hong Kong indicates that the shaft friction for driven piles is always difficult to predict. There is no empirical correlation of shaft friction with SPT $N$ values that is generally applicable to all sites in Hong Kong. Whipping of the pile during driving will often cause a void to be created around the top portion of the pile. As a result, the shaft friction mobilised at the top portion of the pile is usually quite small and not related to the SPT $N$ values of soils. Any attempt to predict the shaft friction of driven steel H-piles in Hong Kong will at best produce a crude estimate.

In this discussion, the writer would like to propose a simple alternative method for estimating the effective length of the pile and determining the final set table based on the energy transfer ratio measured by PDA tests. Using the same symbols as in the author's paper, the energy transferred to the pile and the driving head after impact, denoted by $E^{\prime}$, can be written as

$$
E^{\prime}=\eta f E_{h}
$$

According to the conventional pile-driving formula (that is, equation (1) of the author's paper), the energy transferred to the pile and driving head can be divided into three components as follows:

(a) energy stored in the driving head due to elastic compression, $R_{\mathrm{u}} c_{1} / 2$

(b) energy stored in the pile + soil system due to elastic compression, $R_{\mathrm{u}}\left(c_{2}+c_{3}\right) / 2$

(c) work done against pile resistance, represented by $R_{\mathrm{u}} s$.

The first component represents the energy transferred to the driving head, and the second and third components constitute the total energy transferred to the pile. As the elastic compression of the driving head is usually small compared with the elastic compression of soil and pile, the energy stored by the driving head will be small and can be ignored without making a significant error. As a good approximation, $E^{\prime}$ can be taken as the energy transferred to the pile, denoted by $E_{\mathrm{p}}$, which can be measured directly by a PDA test. If this is the case, equation (23) can be rewritten as

$$
X \approx \eta f
$$

where $X=E_{\mathrm{p}} / E_{\mathrm{h}}$. The magnitude of $X$ is usually given as a standard output of a PDA test.

For a given pile-driving system, the hammer efficiency is usually known. Alternatively, the hammer efficiency can be evaluated by measuring the velocity of the hammer before impact. The variability of hammer efficiency $f$ is usually small in comparison with the uncertainty associated with $X$. Having determined suitable design values of $X$ and $\mathrm{f}$, the effective length, $L_{\text {eff }}$, of a pile can be calculated using the Hiley formula:

$$
\frac{X}{f}=\eta=\frac{W_{\mathrm{h}}+\left(w L_{\mathrm{eff}}+W_{\mathrm{r}}\right) e^{2}}{W_{\mathrm{h}}+\left(w L_{\mathrm{eff}}+W_{\mathrm{r}}\right)}
$$

where $W_{\mathrm{h}}$ is the weight of the hammer, $W_{\mathrm{r}}$ is the weight of the driving head, $w$ is the weight per unit length of the pile, and $e$ is the coefficient of restitution. The above procedure seems to provide a simpler method than that of the author for determining the effective length of driven piles.

Experience in Hong Kong indicates that the energy transfer ratio, $X$, for a site with long piles is governed largely by ground conditions and the characteristics of the hammer. Although the energy transfer ratio, $X$, can vary significantly between sites, the variability of $X$ within a given site is usually small, typically of the order of 5\%. Fig. 4 shows some typical results of measured values of $X$ plotted against the pile length of steel H-piles for a few sites in Hong Kong. It can be observed that the magnitude of $X$ shows no apparent correlation with pile length. This is to be expected for a site with long piles, because the value of $\eta$ remains unchanged if the pile length exceeds the effective pile length.

To implement the proposed method, PDA tests can be carried out for selected working piles of a site. Given the relatively small variability of $X$, a few PDA tests will usually be sufficient to provide reliable estimates of the average value and coefficient of variation of $X$. A suitably conservative value of $X$, say the lower $90 \%$ confidence limit, can be selected as the design value. The effective pile length can thus be determined using this design value of $X$, and the final set table can be prepared accordingly. For a given site where the pile length at final set is longer than the effective pile length, the final set table can be prepared directly using the design value of $X$ without calculating the effective pile length.

The use of PDA tests is not considered to be a handicap for the proposed method as PDA tests can be carried out at relatively low cost. The author's method requires knowledge of the maximum stress induced during pile-driving. Such information will also require PDA tests to be carried out.

Finally, there appear to be a few typographical errors in the author's paper. $E$ should be replaced by $E_{\mathrm{h}}$ in the definition of maximum hammer energy after equation (1). $l_{\mathrm{m}}$ 


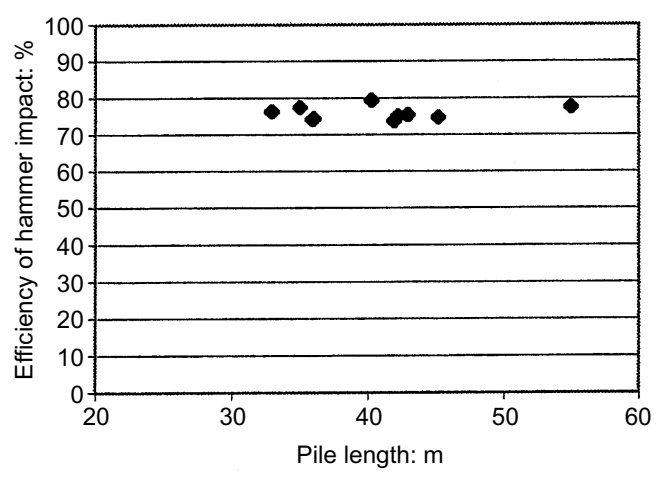

(a)

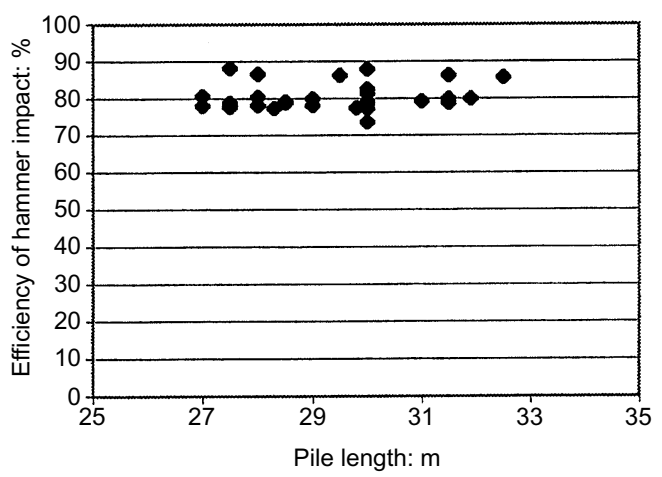

(c)

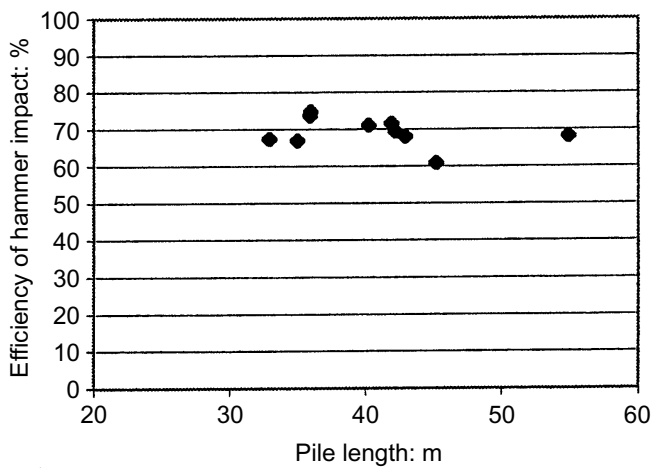

(b)

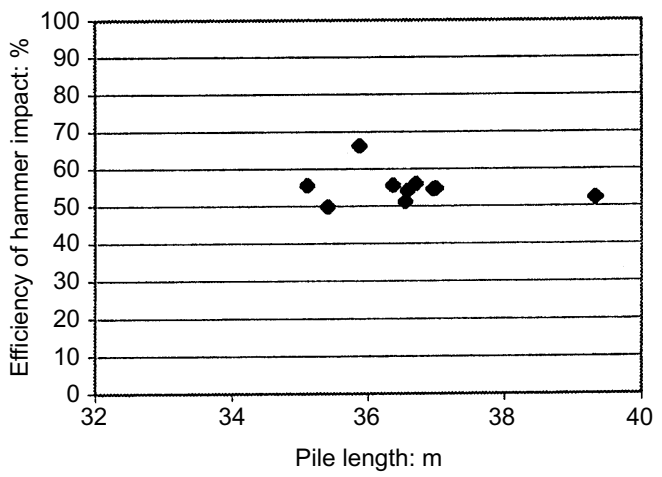

(d)

Fig. 4. Variation of efficiency of hammer impact with pile length for various sites: (a) site A, hydraulic hammer; (b) site A, drop hammer; (c) site B, drop hammer; (d) site C, drop hammer

should be defined as $l_{\mathrm{m}}=m_{\mathrm{H}} / A \rho$ after equation (12a). $c t-2_{0}$ in equation (15) should read $c t-2 x_{0}$. It also appears that the gravity term is missing from the boundary condition of equation (10), because the external forces acting on the hammer include both the force due to gravity and the reaction from the pile.

\section{Author's reply}

The author wishes to express his thanks for the discussion of $\mathrm{Mr} \mathrm{K}$. S. Li and especially for his comments on the typographical errors in the original paper. For corrections the interested reader is also referred to Triantafyllidis (2001). In order to clarify the advantages of the formula proposed in the paper it is worth stating that the assumptions mentioned in the discussion are not absolutely necessary:

(a) the maximum compressive stress, $\sigma_{0}$, induced at piledriving

(b) the estimation of the average shaft friction along the pile

(c) $\varepsilon=1$.

This is because

(a) the impact velocity of the piston can preferably be used if available instead of the stress

(b) the shaft friction with depth and the discretisation length $x_{0}$ are not required to be constant over the entire pile length

(c) assumptions other than $\varepsilon=1$ are also possible.

Further, it is of interest to note that the choice of $\varepsilon$ and $x_{0}$ can be verified by the measured results: that is, the ratio $\varepsilon$, representing the relation between effective pile weight and ram weight, and the choice of $x_{0}$ can be checked by the condition $\sigma=0$ at the time when pile and ram are separated. The discusser concentrates mainly on the examples given in the paper where the data have been extracted from the PDA or dynamic load tests on the test piles PP1 and PP2. The reason for this particular data selection in the paper is that the test results presented by Lam et al. (1994), and especially the values of the shaft friction, have been verified by fully instrumented pile loading tests, and in such cases the empirical correlation between shaft friction and SPT 'values' is not necessary. Nevertheless, the procedure as outlined in the paper represents quite a useful tool for sensible quality control on site. The rational design of driven piles is based on principles of soil mechanics, on the site investigation logs, and on pile load testing. In cases where, on site or close to it, empirical correlations between SPT values, soil formations and skin friction values due to static loading tests are known, it is helpful to use the method outlined in the paper as an estimate and for site control purposes.

Whipping of the pile at the top during driving can be controlled on site, and should be avoided. In cases where the material close to the pile top is non-cohesive, some densification may occur during driving, and the small friction on the top of the pile can represent the lowest possible packing of such material. Nevertheless, the top portion plays only a minor role here. Even the assumption of a linear skin friction distribution with depth at this portion of the pile will not affect the derived effective pile length, because the skin friction will be weighted over the length $l_{\mathrm{m}}$. In addition, it is normal practice in Hong Kong for cut-off levels of the piles normally to appear well below ground level $(2 \mathrm{~m})$ and therefore a follower is used, resulting in the fact that the first metres do not contribute to the pile capacity and therefore they are not taken into account. But even in cases where a follower is not used, the very long piles and the development of the friction over the length are far more important to the behaviour than the first metres. 
With regard to the discusser's comments on the simplified method for determining the so-called effective length, the author considers that this procedure is an oversimplification of the problem under investigation. The magnitude $X$ as given in the PDA tests includes significant parts of energy, which represent the wave attenuation in the ground; this is not considered in the Hiley formula. The very simple formula (equation (25)) proposed by the discusser does not account for ground conditions, and this is obviously in contradiction of the Hong Kong experience.

Moreover, the elastic properties of the system according to equation (25) are not taken into account, where part of the energy is also transferred. For very long piles in particular, the elastic energy increases if the length of the pile is taken instead of the effective length.

The influence of ground conditions on the capacity of piles is clearly shown in the paper of Lam et al. (1994), and it is also clear that the effective length for both piles PP1 and PP2 is not the same even at the same construction site, indicating that $X$ can vary significantly within the site. Figure 4 presented by the discusser shows that the value of $X$ remains more or less constant regardless of the pile length, especially for long piles. In the figure the overall efficiency of the hammer impact is presented. These results are also in agreement with this author's conclusions, because in cases where the real pile length is greater than the effective pile length such a correlation with the efficiency of the impact does not exist. A comparison of the discusser's simplified method with the data of Lam et al. (1994) is missing, and it would be helpful if the discusser could demonstrate the validity of his results, comparing the results with the fully instrumented pile tests on PP1 and PP2. The selection of a few piles where PDA has to be carried out in order to establish an average value of $X$ and a coefficient of variation of $X$ or the calculation of the effective pile length is the way proposed by the discusser. The opinion of the author is that the principles of soil mechanics have to be applied as stated in the paper for determining the effective pile length. Experience gained by instrumental pile load tests on the site or in the vicinity of the site can be implemented in such a rational way that the affected pile length can be estimated during impact. The formula in the paper also works for pile lengths longer than the 'effective' pile length, and the Hiley formula can be applied readily for the final set calculations as a site control measure for every pile without needing to neglect the elastic compression of the system, which will be significant for pile lengths longer than the effective length. The author's proposal can be applied to every type of hammer (hydraulic, drop, diesel) even without PDA tests because a knowledge of the maximum stress induced by the pile during driving is not absolutely necessary. The impact velocity is of importance, and it can be determined directly from the drop height (drop hammer), blow count (diesel hammer) or piston velocity (hydraulic hammer) without knowledge of the stress amplitude induced in the pile.

\section{REFERENCES}

Cornfield, G. M. (1961). Discussion on 'Pile-driving analysis by the wave equation'. J. Soil Mech. Found. Div., ASCE 87, No. SM1, $63-75$.

Geotechnical Engineering Office (1996). Pile Design and Construction. GEO publication No. 1/94, Civil Engineering Department, Hong Kong.

Triantafyllidis, T. (2001). A modification of the Hiley formula for driving long piles and comparisons with test results in Hong Kong. Proc. 14th SE Asian Geotech. Conf. Hong Kong 1, 471-476. 